\title{
User-Friendly Interactive Chinese Character Education System and Its Effect
}

\author{
Yusuke Shimizu ${ }^{1}$ and Jungpil Shin ${ }^{2}$ \\ ${ }^{1,2}$ School of Computer Science and Engineering, The University of Aizu, Fukushima, 965-8580, Japan
}

\begin{abstract}
Background/Objectives: This paper presents a novel friendly and interactive Chinese character (Kanji in Japanese) learning system to enable elementary school students and foreign people living in Japan and to learn Kanji by an interesting and efficient way. Methods/Statistical analysis: By interacting with the network between the client application for students and the server application for teachers, teachers can grasp circumstances of education of all students in real-time. Findings: When students select and input characters they want to learn, the client application checks whether each stroke is unbalanced or not, and it is corrected if unbalanced. If there are no unbalanced strokes, a "Hanamaru" is displayed. On the other hand, the server application has two kinds of display modes, to display circumstances of all students and to display detail circumstances of each student, and teachers can switch them anytime. At the same time, the server takes statistics of points where each student is easy to make mistakes and saves them. So teachers can grasp circumstances and trend of all students. Moreover, pen speed and segment division are introduced in order to extract and correct local features, such as "tome", "hane", and "harai", which were a weak point of previous system in spite of the important point in Kanji education. Improvements/Applications: Segment division is a method that refraction points of each stroke are extracted and each stroke is subdivided by these points and is used to correct "hane" and to distinguish between "ore" and "mage". For both of them, the correction of local features can be realized and the close correction is enabled. Finally, by using the system actually, this system is evaluated whether it is useful to be able to write and memorize Kanji correctly and the interface is really friendly for learners. As a result, since many students feel interesting and useful to learn Kanji, it is clear that this system is useful for Kanji education. And the interface is also a high appraisal.
\end{abstract}

\section{Index Terms}

Character Education, Handwriting, Human Computer Interaction, User-friendly.

\section{Corresponding author : Jungpil Shin}

jpshin@u-aizu.ac.jp

- Manuscript received July 13, 2017.

- Revised August 4, 2017; Accepted September 1, 2017.

- Date of publication September 30, 2017.

(1) The Academic Society of Convergence Science Inc.

2546-1583 @ 2017 IJEMR. Personal use is permitted, but republication/redistribution requires IJEMR permission. 


\section{INTRODUCTION}

Since thousands of Kanji, in addition to Hiragana and Katakana, are used in Japan, it is a essential to master them in primary education. However, since it is hard to master enormous Kanji, it is important to master them little by little for every grade. Hence, it takes six years to learn Kanji for education (about 1000 characters), and three more years to learn designated for everyday use (shown in Fig. 1). Yet as it stands, it is difficult to have an enough education in the limited time, such as classes, because the number of classes is reduced.

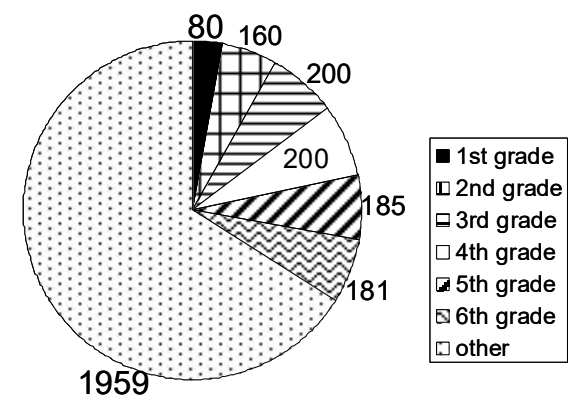

Fig. 1. breakdown of the number of Kanji which can learn with my system

On the other hand, foreign people living in Japan are very increased along with the economic globalization. Of course, it is essential for them to read and write Kanji, too. However, it is also not easy for them to master Kanji in a short time as well as Japanese. Especially, it is very hard for those who use European roundish languages to learn Kanji by themselves which emphasize various features, such as stops, bounding, relative length, and so on.

Moreover, since there are various acquirement degree and attention for each student, it is great load for one teacher to teach courteously to all students. Hence, software that cannot only aid learners in mastering them efficiently and in a short period but also be learned anytime at home and at school is needed.

For this, research and development of Kanji education systems have been carried out by various styles. For example, Tatsuoka and Yoshimura have developed the Kanji education support system intended for foreign people and elementary school students [1]. Since this system has a graphical interface, it is easy to use it. Takesue has developed a system enabling teachers to designate evaluation points of character shapes [2]. This system has functions of not only evaluating stroke orders and character shapes but also enabling teachers to designate evaluation points and replace the model of character patterns easily and freely. Therefore it is considered in not only learners but also teachers. Yamazaki has developed a system based on the analysis of pen speed [3]. This system not only corrects character shapes and stroke orders but also has dynamic display of pen movement for learners to understand a change of pen speed instinctively. Yamamoto has developed a CAI system using calligraphic skill knowledge [4]. This system has various skills such as stroke order, the shape of characters and strokes, and arrangement of radicals as knowledge of an educational tool. Moreover, wrong skill knowledge is prepared for identifying and correcting mistakes. In addition, systems considered so that learners can master Kanji via games have also been developed [5][6]. Otherwise, various games with which players can learn Kanji without circumstance with a cellular game player are developed and popular.

The model of Kanji education system I have ever studied is to correct strokes by comparing reference characters with input characters using inter-stroke information mentioned later. However, since this comparison is fulfilled at five points estimated equally from each stroke, this model had a weak point that cannot catch local features.

Then, by introducing pen speed and segment division, this system is improved so that it can catch them. Pen speed is a speed of writing characters. Segment division is a method that refraction points of each stroke are extracted and each stroke is subdivided by these points. For both of them, the correction of local features can be realized and the close correction is realized. In addition, the server application is developed and the novel function of the network communication realizes better interactive system.

\section{RELATED STUdies}

This system is based on several algorithms, such as real-time search of relation with correct stroke by Cube search [7], modification of a letter-shape using inter-stroke information, and instruction of the order of writing using animation [8], and setting a bar which can freely set a threshold which judges whether each stroke should be corrected or not [2].

\section{A. Stroke Correspondence Search}

I An input character is expressed as an ordered series of strokes as follows:

$$
A=A_{1} A_{2} A_{3} \cdots A_{k} \cdots A_{N}
$$

where the $k$-th stroke $A_{k}$ is the time sequence representation of local feature $a_{i k}$ of a character, e.g., $\mathrm{x}-\mathrm{y}$ coordinates or stroke direction are expressed as

$$
A_{k}=a_{1 k} a_{2 k} \cdots a_{i k} \cdots a_{I k}, \quad I=I(k)
$$

The reference pattern is similarly expressed as

$$
B=B_{1} B_{2} B_{3} \cdots B_{k} \cdots B_{N}
$$

and

$$
B_{l}=b_{1 l} b_{2 l} \cdots b_{j l} \cdots b_{J l}, \quad J=J(l)
$$


where $N$ is the number of strokes of a character.

One-to-one stroke correspondence is defined by bijection $l(k)$ to stroke number $k$ of the input pattern. The measure of dissimilarity between the input pattern stroke $A_{k}$ and the reference pattern stroke $B_{l}$ is calculated using stroke information on the shape and position. The measure of dissimilarity is defined as $\delta(k, l)$ and called the stroke distance.

The sum of the distance $\delta(k, l)$ between strokes is used as a valuation basis of the optimal correspondence. The minimum value $D(A, B)$ is calculated by the following formula, and the stroke correspondence $l(k)$ is obtained as the following result:

$$
D(A, B)=\min _{\{l(k)\}}\left[\sum_{k=1}^{N} \delta(k, l(k))\right]
$$

These processes for all stroke distances require an amount of calculation of the factorial order of the number of strokes and are unreal. Then, the 3rd Markov cube is used by the result that 3rd is enough from preliminary experiment [3]. Furthermore, the amount of calculation is decreased for the index order from the factorial order using a beam search. So the stroke correspondence search in real time is possible, and the wrong order of a stroke can be pointed out precisely.

\section{B. Inter-Stroke Information}

As a geometric feature of a character, a relative arrangement relation exists between strokes. Moreover, the relative merits and demerits between strokes can become as important work for discernment between some characters (Fig. 2). Such information is defined as inter-stroke information.

The vector connects the $i$-th representation point of stroke $k$-th of an input pattern and the $j$-th representation point of stroke $p$-th as

$$
d_{i j}\left(A_{k}, A_{p}\right)
$$

The vector connects the $i$-th representation point of stroke $l$-th of a reference pattern and the $j$-th representation point of stroke $q$-th as

$$
d_{i j}\left(B_{l}, B_{q}\right)
$$

The Inter-stroke information $\rho$ is expressed following formula,

$$
\rho(k, l ; p, q)=\sum_{i=1}^{m} \sum_{j=1}^{m} R\left(d_{i j}\left(A_{k}, A_{p}\right), d_{i j}\left(B_{l(l)}, B_{l(q)}\right)\right)
$$

where $\mathrm{m}$ is the number of the representation point, and $R(\cdot, \cdot)$ are the weighted sums of the angle difference $(\theta)$ of vector and the difference $(e)$ of the length of a vector.
The inter-stroke information is considered as a measure of dissimilarity of the position relation of each stroke of the input pattern and the position relation of each stroke of the reference pattern.

The relative position relation with the representation point of other strokes is the one of the evaluation values by $m=5$, i.e., five points that are equally estimated from each stroke. Therefore, this system cannot catch local features, such as "tome", "hane", and "harai".
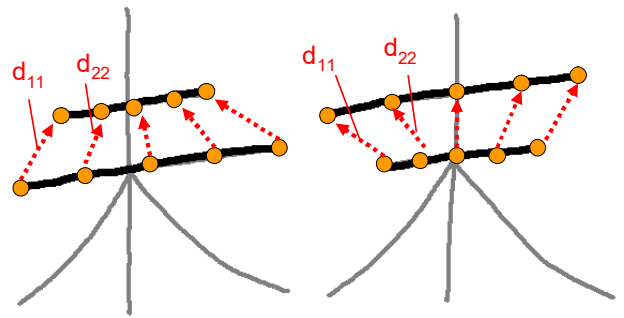

Fig. 2. Inter-Stroke Information

\section{Instruction of the Character Using Inter-Stroke Information}

The inter-stroke information uses as a valuation basis, and the evaluation value $\rho$ is considered which the stroke of a larger value than a threshold breaks down the character. Such a stroke is corrected by the instruction. $k^{\prime}$ is the drawing to which $\rho$ becomes larger than the threshold, and $k^{\prime}-1$ is the stroke in front of $k^{\prime}$. The number of a representation point is set to 5 , the vector

$$
d_{11}\left(B_{k^{\prime}-1}, B_{k^{\prime}}\right), d_{22}\left(B_{k^{\prime}-1}, B_{k^{\prime}}\right), \cdots, d_{55}\left(B_{k^{\prime}-1}, B_{k^{\prime}}\right)
$$

is displayed in piles on an input pattern as correction directions.

For example, two characters shown in Fig. 3 instruct to correct the relational position of the 1st stroke and the 2 nd stroke. $d_{11}$ is the vector which connects the 1 st representation points of the stroke and $d_{22}$ is the vector which connects the 1 st representation points of the stroke.

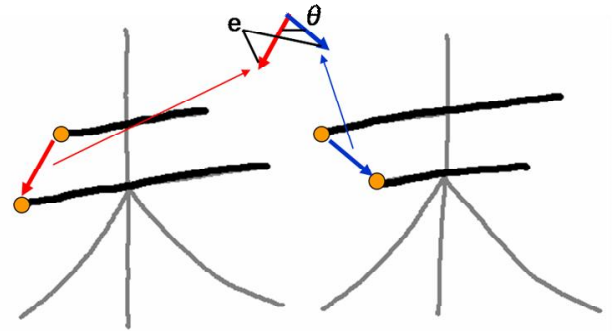

Fig. 3. Instruction of the Character Using Inter-Stroke Information

\section{Correction Threshold}

When characters are corrected, strokes which should be corrected are determined and character instruction is performed using a correction threshold $d$. Therefore, the number of correction strokes changes. 
Each characteristic point of reference and input strokes are subtracted and calculated as absolute value, and threshold $d$ is calculated using the following formula.

$$
\begin{gathered}
d=\frac{s+m+e}{3} \\
s=\frac{\left|s(x)_{r e f}-s(x)_{i n p}\right|+\left|s(y)_{r e f}-s(y)_{i n p}\right|}{2} \\
m=\frac{\left|m(x)_{r e f}-m(x)_{i n p}\right|+\left|m(y)_{r e f}-m(y)_{i n p}\right|}{2} \\
e=\frac{\left|e(x)_{r e f}-e(x)_{i n p}\right|+\left|e(y)_{r e f}-e(y)_{i n p}\right|}{2}
\end{gathered}
$$

where $s, m$, and $e$ is characteristic points of each stroke (That is, starting point, middle point, and end point), $x$ and $y$ is $\mathrm{x}-\mathrm{y}$ coordinates, ref and inp are reference and input strokes.

\section{KANJI EdUCATION SYSTEM}

In this section, the interface of this system is mentioned.

This system is designed so that it can score automatically and display it to evaluate objectively. Moreover, since this system is based on the previous system, its animation display [5] and Hanamaru display [9] are also included in this system.

By the way, in this system, learners can master the first 2,965 characters of JIS (Japan Industrial Standard) including Kanji designated for everyday. By operating buttons, a study range can be chosen from following levels: first to sixth grade of elementary school, Kanji for education, and the first level of JIS include them. But learners cannot learn Hiragana, Katakana, Alphabets, and figures because this system targets only Kanji.

Learners input characters with a pen tablet (WACOM KT-0405-RN) as an input device. And, the data of $\mathrm{x}$ and $\mathrm{y}$ coordinates are extracted from the tablet at regular time intervals and input strokes are displayed in the system. For this, learners can write checking the shape, the size, and the order of the character.

\section{A. Interface}

The interface of this system is shown in Fig. 4.

(a) The network between the teacher and the student is connected.

(b) The study range is changed. In "EDU" mode, 1006 educational Kanji learned in the elementary school can be learned. And In "JIS" mode, 2965 Kanji including Kanji designated for everyday use can be learned. (c) The language of instruction to either Japanese or English is changed. The English mode is considered so that even foreign people can learn easily.

(d) This system is exited.

(e) The character learning now is displayed.

(f) Learners write the character here with pen and tablet. The correction result is also displayed here.

(g) Another character is displayed on the reference character column using the "Back", "Next", and "Random" buttons. A stroke correspondence table is expressed as a "Check" button, and an animation is expressed as the "Anime" button.

(h) An animation is displayed.

(i) The incorrect input stroke orders and a score of input character are displayed. If there is wrong stroke order, the color of wrong strokes changes. Without any wrong order, the score of input character is displayed.

(j) A stroke correspondence relation is displayed.

(k) By inputting the character with the keyboard into this box, learners can quickly select to any character they want to learn.

(I) Within the range list, learners can select to any character they want to learn.

(m) By threshold bars, learners can change the level of character instruction. When set to "STR", the judgment of character instruction becomes strict and most characters are corrected. When set to "LSE", the judgment of character instruction becomes loose and only the strokes which should be corrected are done according to desired thresholds.

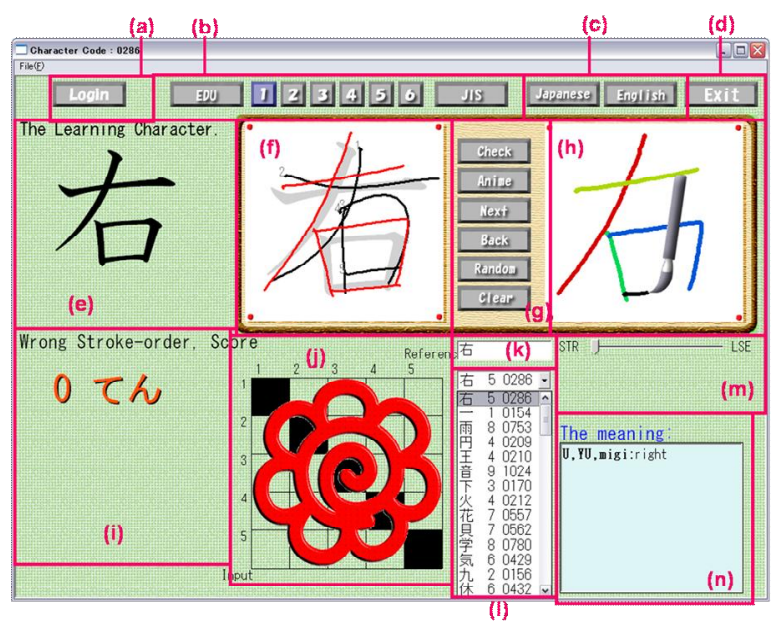

Fig. 4. Interface of Kanji Learning System: (a) Login button, (b) Study range selection button, (c) Language change button, (d) Exit button, (e) Reference pattern, (f) Character input column, (g) Operation button, (h) Animation display column, (i) Wrong stroke order and score display column, (j) Stroke correspondence table, (k) Character input box, (1) Character list, (m) Threshold bar, (n) Meaning display character column 
(n) The meaning of learning character is displayed. Japanese and English definitions are prepared.

\section{B. Animation Display}

Learners can understand the order of strokes virtually by animation. By changing colors of each stroke used in the animation, learners can understand virtually not only the order of strokes by animation but also where the same strokes are from and to. In addition, colors are limited to 12 colors because of the small difference in colors (Fig .5).

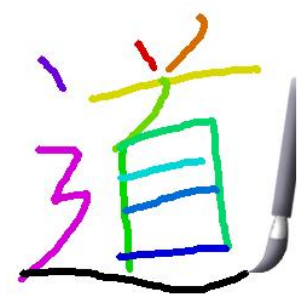

Fig. 5. Stroke Colors in Animation

\section{Score and Hanamaru Display}

This system is improved so that it can grade based on DP value mentioned, the number of corrected strokes, and mistakes of local features and can display the score to evaluate objectively. In a perfect score, a Hanamaru displays on the input character shown in Fig. 6. The aim is that the score and Hanamaru displays bring learner's will for learning. This score is also displayed on the server and stored every time through the network.

Fig. 6. Display of Hanamaru

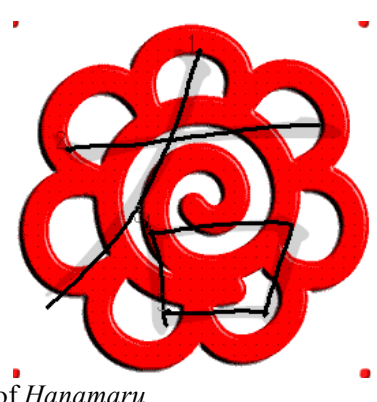

\section{Novel Correction Algorithm}

This system had a weak point so far that it cannot catch local features. But it is very important to understand and master these features correctly in the Kanji learning. Then, the method of correction is improved in this paper. Here, some examples of local features are shown in Fig.7. For the improvement, two following algorithms are introduced.

1. pen speed

2. segment division

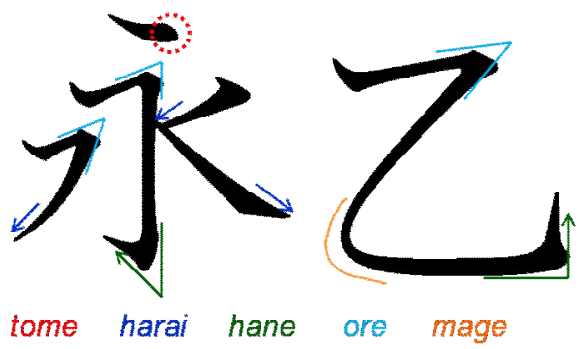

Fig. 7. Examples of local features

\section{A. Flow of Correction}

The flow of character correction is shown in Fig. 8.

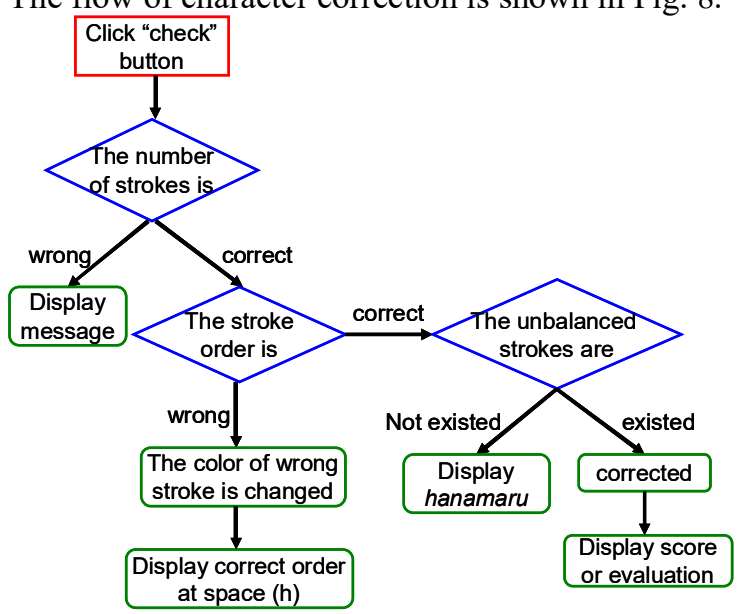

Fig. 8. Flow of Correction

When learners click the "Check" button of the operation button after writing a character, the stroke correspondence between the reference character and input character is examined at first. If the number of strokes is wrong, a message window is displayed. And if the order of strokes is wrong, colors of wrong strokes change and the correct order is shown. Without any mistake at stroke numbers and stroke orders, the character is corrected and the score is displayed.

\section{B. Pen Speed}

If you just write Kanji casually, a difference between tome and harai does not seem so important because the difference is hard to distinguish with the appearance in the case of writing with a pencil unlike a brush. But it is very important to distinguish them to be able to write balanced characters carefully and smoothly. Therefore, this system is improved them to be able to do them by using the pen speed.

The maximum values of the pen speeds of the $k$-th reference and input strokes are defined as $v(k)_{\max }$ and $v^{\prime}(k)_{\max }$, the pen speeds of the $k$-th reference and input strokes at the end points are defined as $v(k)_{\text {end }}$ and $v^{\prime}(k)_{e n d}$, and the pen speeds of the $k$-th reference and input strokes at the begin points are defined as $v(k)_{\text {begin }}$ and $v^{\prime}(k)_{\text {begin }}$ respectively. Then, if following 
conditions $C_{k}, C^{\prime}{ }_{k}$ are true, the $k$-th stroke is judged as harai and if false, the stroke is judged as tome.

$$
\begin{gathered}
C_{k}: \frac{v(k)_{\text {end }}}{v(k)_{\max }} \geq 0.6 \text { and } \frac{v(k)_{\text {end }}}{v(k)_{\text {begin }}} \geq 2.5 \\
\text { for the } k \text {-th reference stroke, } \\
C_{k}^{\prime}: \frac{v^{\prime}(k)_{\text {end }}}{v^{\prime}(k)_{\max }} \geq 0.6 \text { and } \frac{v^{\prime}(k)_{\text {end }}}{v^{\prime}(k)_{\text {begin }}} \geq 2.5 \\
\text { for the } k \text {-th input stroke. }
\end{gathered}
$$

This judgment is carried out for all reference and input strokes. If $C_{k}$ does not coincide with $C^{\prime}{ }_{k}$, the $k$ th stroke is corrected.

\section{Segment Division}

Segment division is introduced in order to check whether hane is existed and it is appropriate length or not for strokes having hane and whether there is a mistake of ore and mage for strokes having them. Here, segments are parts which each stroke is subdivided into at points where it folds, called refraction points. A method of the segment division is shown in Fig. 9.

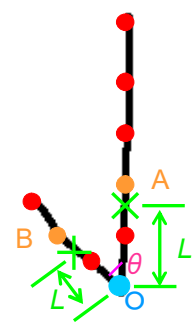

Fig. 9. A method of the segment division

A certain point on a certain stroke is defined as $\mathbf{O}$. A point $\mathbf{A}$ is defined as a point written earlier than $\mathbf{O}$ and the distance between $\mathbf{A}$ and $\mathbf{O}$ is longer than $L$. A point $\mathbf{B}$ is defined as a point written later than $\mathbf{O}$ and the distance between $\mathbf{B}$ and $\mathbf{O}$ is longer than $L$ (Here, $L=15$ in this division). And an angle made by $\mathrm{OA}$ and $\mathrm{OB}$ is defined as $\theta$. Then, if $\theta$ is larger than $\alpha, \mathbf{O}$ is defined as a refraction point (Here, $\alpha=120$ degree in this division). This process is carried out for all points of each stroke. Here, if refraction points are found continuously, a point whose value of $\theta$ is minimum is only defined as a refraction point and other points are removed. Finally, strokes are divided with these refraction points and segments are generated.

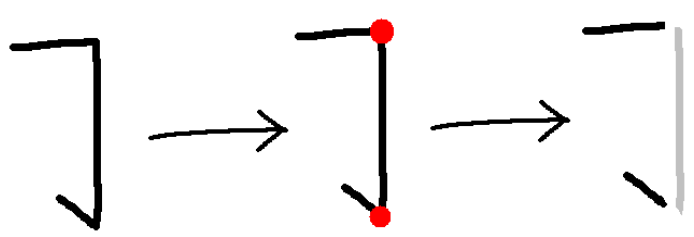

Fig. 10. An example of the segment division (Two red circles are refraction points.)
An example of the segment division is shown in Fig. 10.

In this case, the stroke is divided into three segments by two refraction points (circles in Fig. 10). Moreover, there are some cases that only one segment is existed in one stroke, such as dots and straight strokes.

This division process is carried out for both reference strokes and input strokes. If the number of segments of a reference stroke coincides with that of an input stroke, the stroke is corrected. And if the length of the segment is extremely wrong, it is also corrected. For this, the correction of hane is greatly improved.

\section{Correction Result}

The correction result is shown in Fig. 11.

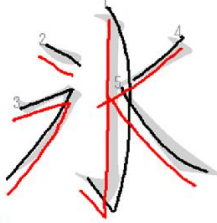

(a)

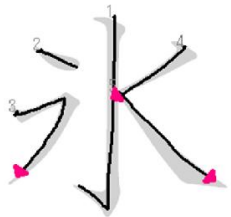

(c)

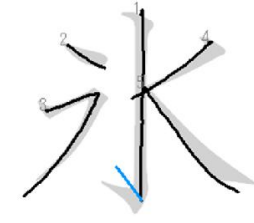

(b)

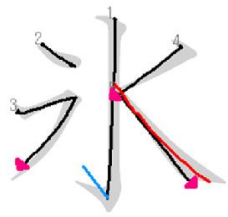

(d)
Fig. 11. Correction Result: (a) is a case that all strokes are corrected, (b) is a case that only hane is corrected because of no hane. So a blue stroke is added. (c) is a case that strokes should be harai are tome. And (d) is a case that all strokes are tome in spite of harai or hane. Here, triangle marks mean that these strokes should be harai and when learners write them with tome by mistake, these marks are displayed.

\section{Client-Server System}

In this section, the novel network communication constructed in this paper is mentioned about. This system is improved to be able to interact with the network communication between the client application for students and the server application for teachers. For this, teachers can grasp circumstances of learning of all students in real-time.

\section{A. Client Application}

A new button "Login" is added on the client application to realize an interactive system. When students click this button, a dialog shown in Fig. 12. When they enter some personal information and password correctly, they can login to the server. In addition, by clicking "Login" button again, students can logout. 


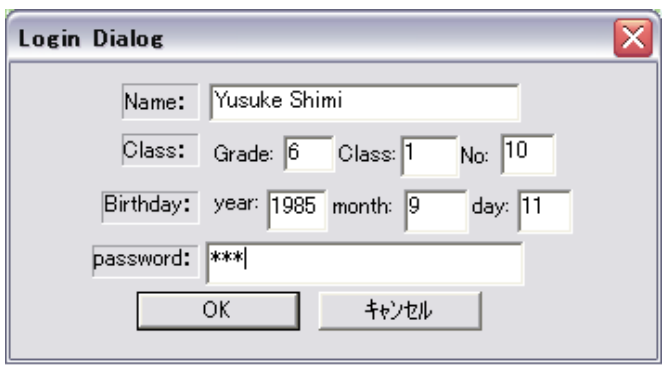

Fig. 12. Login Dialog

\section{B. Server Application}

The server application has two kinds of display modes and teachers can switch them anytime. So teachers can grasp current circumstances, current detail circumstances, and statistics of all students in real-time.

\section{1) Class Mode}

The interface of the class mode is shown in Fig. 13. In this mode, circumstances of all students are displayed.

(a) (b) (c)

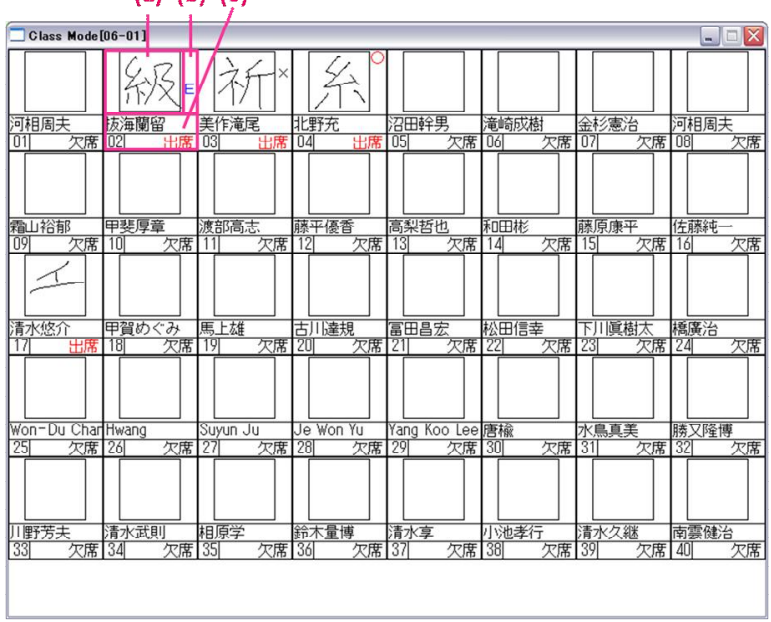

Fig. 13. Class Mode (a) Stroke display column, (b) Judgment display column, (c) Personal information display column

(a) Strokes written by each student are displayed in real time.

(b) A judgment of input characters is displayed. There are 3 kinds of judgments, and the circle shows no correction, cross shows some or all strokes are corrected, and "E" shows there is wrong stroke order or the number of strokes.

(c) The personal information of the focused student, such as his name, number, attendance.

\section{2) Detail Mode}

The interface of the detail mode is shown in Fig. 14. In this mode, detail circumstances of each student are displayed. (a) The personal information of the focused student, such as his name, number, attendance, and his head shot.

(b) Strokes written by the focused student are displayed in real time. After the correction, a score and comment are also displayed and the color of corrected strokes changes.

(c) The current input character is displayed.

(d) Four previous input characters are displayed. In (c) and (d), a judgment, score, and the number of each correction are also displayed.

(e) The class mode is gone back to.

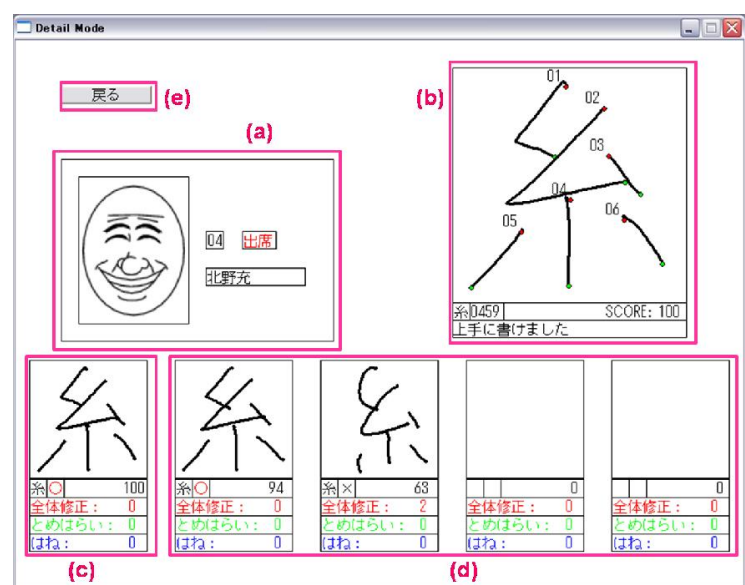

Fig. 14. Detail Mode(a) Personal information display column, (b) Character information display column, (c) Current character display column, (d) Past character display column, (e) Return button

This detail mode takes statistics of points where each student is easy to make mistakes. There are three kinds of statistics, mistakes of tome and harai, hane, and the number of strokes and the order of strokes. These statistics are saved to the server in the reception.

\section{Examples of Interaction}

The example of interaction is shown in Fig. 15.

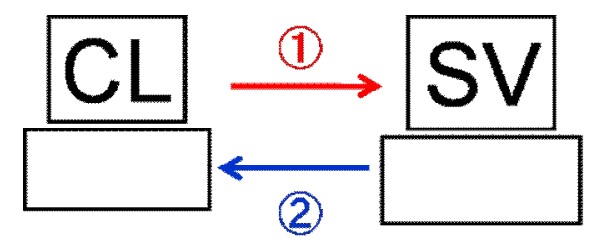

Fig. 15. Interaction between client and server

1) Client -> Server

(a) LOGIN INFORMATION: Send login information and apply to the server for the login.

(b) STROKE DATA: Stroke data students are writing now.

(c) KANJI DATA: Characters students are learning now. 
(d) STATISTIC DATA: Scores and the number of mistakes of each student.

(e) INITIALIZE: The reset of input strokes or change of the learning character.

2) Server $->$ Client

(f) LOGIN SUCCESS / FAILURE: The server informs whether the login from the client is succeeded or failed.

(g) SERVER END / ERROR: Information of stopping connection or some abnormality in the server.

As soon as students finish writing one stroke, data (c) is sent to the server and the stroke is displayed there. Data (e) is sent when students click "Check" button and the score and correction results are displayed. Data (f) is sent when students click "Clear" button and rewrite a character or when they change another character.

\section{Evaluation}

In this section, the evaluation of this system is mentioned about. By using the system actually, the purpose is to confirm whether the system is useful to be able to write and memorize Kanji correctly and whether its interface is really friendly for learners.

\section{A. Method of Evaluation}

Members of my laboratory and foreign students are cooperated for this evaluation. The breakout of members is Japanese with 7 students, Korean with 4 students. Here, this paper defines students from countries where Kanji is not used as foreign students. Characters used in the evaluation are differed among there groups. The list of these characters is shown in Table 1.

Table 1. List OF KANJI USED IN THIS EVALUATION

\begin{tabular}{|c|c|c|}
\hline $\begin{array}{l}\text { For Japanese } \\
\text { students }\end{array}$ & $\begin{array}{c}\text { For Korean } \\
\text { students }\end{array}$ & $\begin{array}{c}\text { For foreign } \\
\text { students }\end{array}$ \\
\hline 爾 細 & 第 逆 延 & 水 学 車 \\
\hline 簊 縜 鹸 & 骨 編 報 & 竹 骨 逆 \\
\hline
\end{tabular}

Since Japanese are used to writing Kanji, five characters seemed it hard to write are selected. Some of them are not Kanji designated for everyday use, which are environed with the square. Moreover, Korean students are used to writing Kanji a little since Kanji is also used a little in Korea and they learned shortly in coming to Japan. So characters which are learned in the high grade of the elementary school and which is easy to mistake the order and the number of strokes are selected. On the other hand, since foreign students are not used to Kanji, four simple characters whose local features and relative length of strokes are important and two characters Korean students write are selected.
The evaluation is carried out by the following flow.

(1) Learners input their login information and login to the server.

(2) They write characters by actually using the client application and practice all characters 15 times per each character shown in Table1.

(3) When learners finish practicing all characters, they answer the questionnaire at last.

(4) The number of corrections and scores are saved to the server every time.

Learners write characters with a pen and tablet display. By using these devices, they can be evaluated in the same style of practicing with a note and pencil.

Next, the content of the questionnaire is:

$<\mathbf{1}>$ Did you feel interesting?

$<2>$ Do you want to use this system again?

$<3>$ Do you think that this system is useful for you to learn Kanji?

$<\mathbf{4}>$ Do you think that this system is useful for you to be able to write balanced Kanji?

$<5>$ Do you feel that this system is interactive?

$<\boldsymbol{6}>$ Do you feel that the result of correction is easy to understand?

$<7>$ Do you feel that this system is a user-friendly interface?

$<\mathbf{8}>$ General evaluation.

$<9>$ Could you select any characters you felt easy to write? (multiple selections)

$<10>$ Could you select any characters you felt hard to write? (multiple selections)

$<$ 11 $>$ Could you select at most 3 functions or operations you felt useful, good, or wonderful?

$<12>$ Could you select at most 3 functions or operations you felt useless, bad, or boring?

In addition, foreign students also answer the period of living in Japan and their hometown.

From $<\mathbf{1}>$ to $<\mathbf{8}>$ of these questions are evaluated by 5 grade scale from 1 to 5 . Questions $<9>$ and $<\mathbf{1 0}>$ are prepared to search which characters for the evaluation are easy and hard to write for learners. Questions $<\mathbf{1 1}>$ and $<\mathbf{1 2}>$ are questions about the interface. User-friendly functions and operations are one of amenities. Vice versa, user- unfriendly ones should be further improved in the future. Moreover, choices are:

$<\mathbf{1}>$ Input login information

$<2>$ English Display Mode (choice except Japanese)

$<3>$ Display of character you are learning now

$<\mathbf{4}>$ Display of model character in character input column

$<5>$ Corrected stroke display

$<\mathbf{6}>$ user-friendliness of operation buttons

$<7>$ Animation display

$<8>$ Wrong stroke order display

$<9>$ Score display

$<\mathbf{1 0}>$ Stroke correspondence table

$<\mathbf{1 1}>$ Character section list 
$<\mathbf{1 2}>\quad$ Meaning display

$<$ 13 $>\quad$ Nothing

\section{B. Questionnaire Result}

In this subsection, questionnaire results are mentioned about. The result of averages from Question $<\mathbf{1}>$ to $<\mathbf{8}>$ is shown in figure 16 .

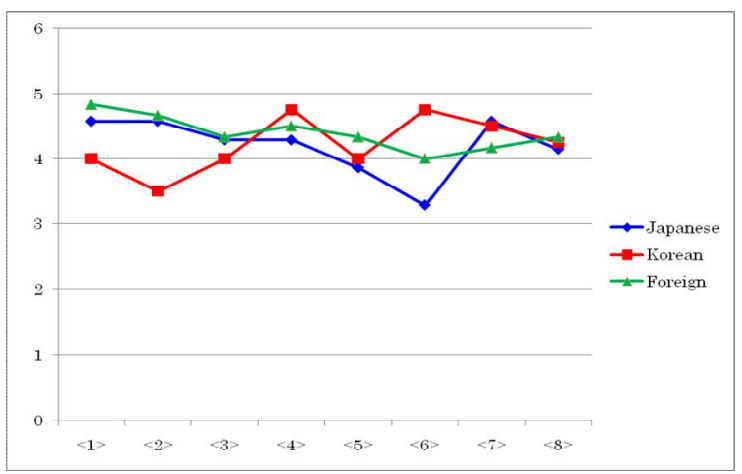

Fig. 16. Results of Averages from Question $<1>$ to $<8>$

Many students feel interesting for using this system and high appraisals for all groups. Moreover, since question $<\mathbf{3}>$ is also a high appraisal, it is clear that this system is useful for Kanji learning. And the interface is also a high appraisal.

On the other hand, Question $<\mathbf{2}>$ is a high appraisal for Japanese and foreign but is not so a high appraisal for Korean. Question $<\mathbf{4}>$ is a higher appraisal for Korean and foreign than that for Japanese, vice versa. It seems that, compared with Japanese, they feel that though they come to be able to write Kanji correctly, they may not be able to memorize Kanji. Because, learners tend to a mechanical learning by only tracing the reference and actually I also feel so through this evaluation.

However, Question $<\mathbf{6}>$ is a higher appraisal for Korean and foreign students than one for Japanese. It seems that foreign students refer rather to correction results so that they will write better or be careful of this part. Meanwhile, it seems that since Japanese students are used to writing Kanji and understand local features, there may be some gaps between their habits and correction results. Anyway, it is necessary to not only improve easier to understand correction results but also show clearly why this stroke is corrected. In addition, it is remarkable that Question $<\mathbf{6}>$ is a higher appraisal for foreign students than one for Japanese and Korean.

The result of totals of Question $<\mathbf{9}>$ and $<\mathbf{1 0}>$ for Japanese, Korean, foreign students is shown in Fig. 17 (a), (b), and (c) respectively.

Compared with Japanese, both characters that Korean and foreign students feel easy to write and feel hard to write vary a little. For instance, many Korean students feel easy to write “骨” because it seems that its shape looks simple. In contrast, many
Korean students also feel hard to write “編” because it seems that it has many strokes and its shape looks complex. At the same time, many foreign students feel easy to write “車” because it seems that it has many straight strokes and its shape looks simple. In contrast, many foreign students also feel hard to write “逆” because it seems that its shape, especially "shinnyo", looks complex.

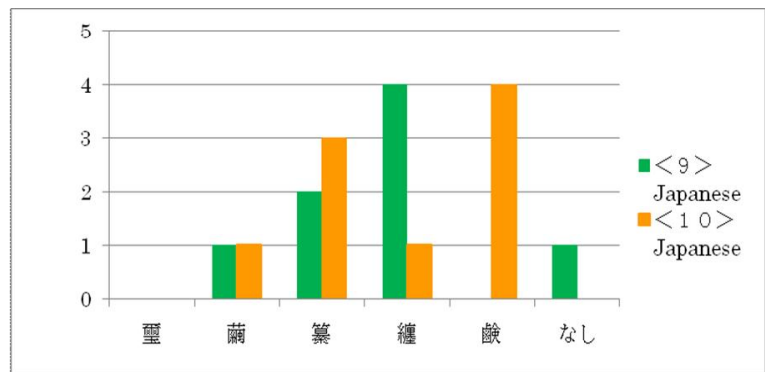

(a)

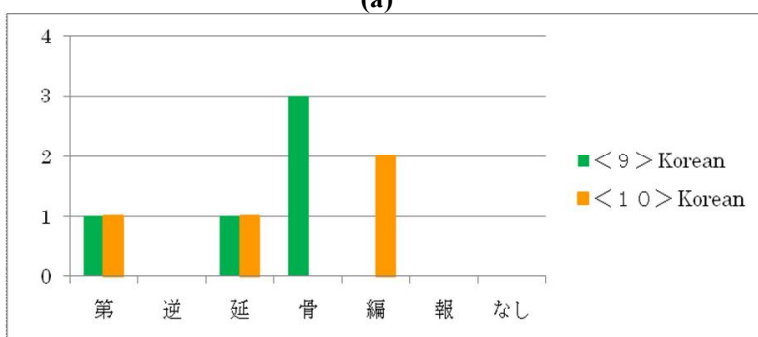

(b)

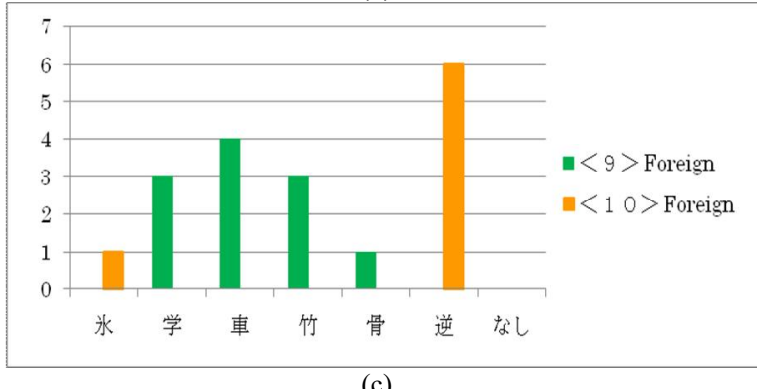

Fig. 17. Result of Total of Question $<9>$ and $<10>$ for (a) Japanese (b) Korea and (c) Foreign

On the other hand, characters vary widely for Japanese because of perhaps individual liking and fortes. Moreover, surprisingly, there are many students who feel easy to write “纏” because it seems that its shape and stroke order may be simple. In contrast, there are many students who feel hard to write “鹸” because it seems that its shape of the left part and stroke order are complex and they may have some burden to write. The result of the total of Question $<\mathbf{1 1}>$ is shown in Fig. 18.

The animation display is a high appraisal for all groups. It is used in characters they write for the first time or which have complex parts such as nyo, dots and crosses of “鹸” and “䨪”. Moreover, wrong stroke order display is also a high appraisal and it 
seems that it is useful in understanding correct stroke order.

On the other hand, the score display is a high appraisal for Japanese as well as animation but is not so a high appraisal for Korean and foreign students. It seems that raising the score is a direct factor of improving the learning eagerness for Japanese but wanting to writing better is the direct factor for the other students rather than raising the score. In addition, since it seems that they are hardly used and remained in the impression, no students select operation buttons or character list. The result of total of Question <12> is shown in Fig. 19.

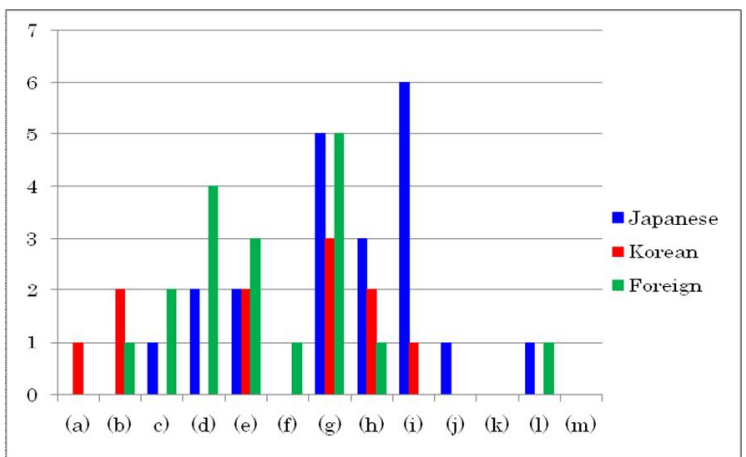

Fig. 18. Result of Total of Question $<11>$

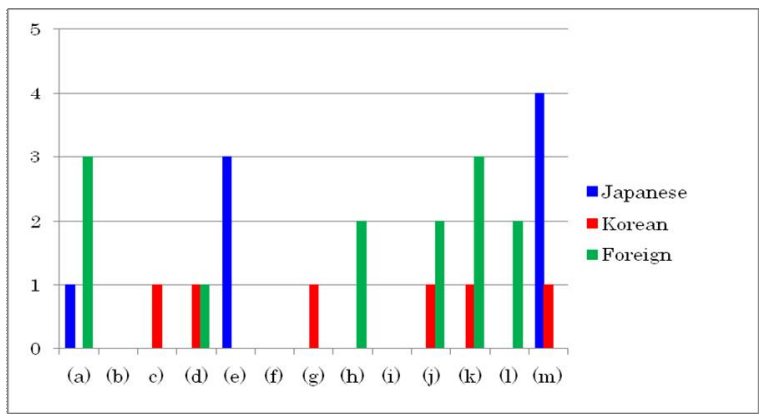

Fig. 19. Result of Total of Question $<12>$

Since three Japanese students selected the correction result, it will be necessary to improve it. However, since most Japanese students answered "Nothing", there are few bad functions for Japanese.

On the other hand, the results of Korean and foreign students vary. Moreover, it is clear that a few foreign students answered "Nothing" and most students have at least one function to improve.

\section{Score Change Result}

Fig. 20 shows average percent changes of scores of all students as the score in the first time is 100 . Totally many characters of ranges of score changing do not vary so much. Especially, the character "鹸” selected as hard to write is seemed that students cannot be used to writing it only 15 times practice and that it may be necessary to learn it for a long period. Moreover, though characters such as “壐”, “編”, and “第” have complex tome and harai, scores of them rise little by little. In contract, the score of character “逆” falls little by little for both Korean and foreign. It seems that since students may be careful of a part "shinnyo", there are some mistakes of harai and strokes at the other part.

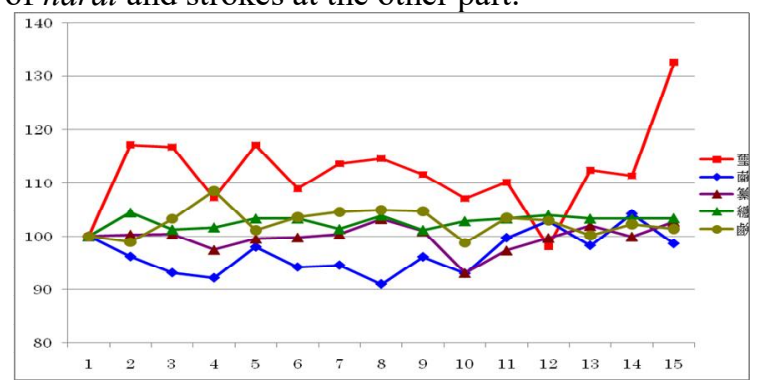

(a)

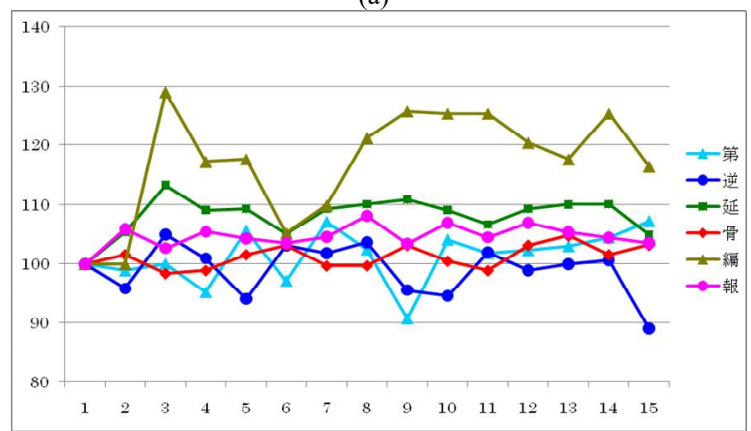

(b)

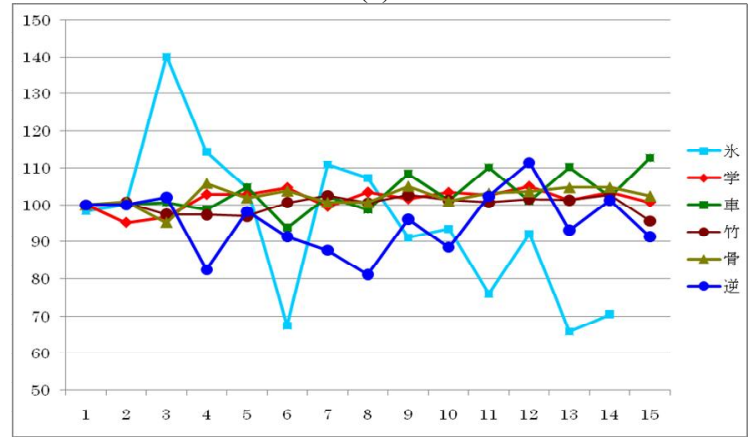

(c)

Fig. 20. Average Percent Changes of Scores (a) is for Japanese students, (b) is for Korean students, and (c) is for foreign students.

\section{DISCUSSION}

Through this evaluation, some problems to be improved are emerged. First of all, though the interaction is a high appraisal, it is necessary to improve it. For instance, there are advices of sending automatically from the server, such as "you should be careful of this part" and "write this part like this example" and a function that teachers can correct directly, this problem will be improved widely. In addition, it is also necessary to show why a stroke is corrected in the correction instructions.

Next problem is the reference character data. 
The reference data used by the current system is all hand-written. However, since there are habits or obsessiveness in handwritten characters, it is almost impossible to make more than 3,000 perfect reference data. And this problem can have a bad influence to the correction results. Then, by using existent font files for the reference data, there may be a method to match between online data of input strokes and offline data of fonts. By this method, there will be no need to make 3,000 perfect reference data.

\section{CONCLUSION}

This paper has presented a method of stroke correction using pen speed and segment division. For both algorithms, the correction of local features can be realized and the close correction is enabled. In addition, the server application is developed and the novel function of the network communication realizes better interactive system. And, evaluation results show constant education effects and a high evaluation of the interface.

From now on, it is essential to improve reference character data. In addition, it is also necessary to enhance functions of the interaction. To develop friendly interfaces for not only students but also teachers should be one of important arguments in the future.

\section{REFERENCES}

[1] Tatsuoka, R., Yoshimura, M. (1996). Development of A Kanji Learning System for Foreign Students or Elementary Students, IEICE Trans. \& System, ET96-36

[2] Takesue, N., Mochida K., Kitadai, A., Nakagawa M., (2005) A handwriting-based Kanji learning system enabling teachers to designate evaluation points, IPSJ SIG SIG Technical Report, 2005-CE-78, 15-22

[3] Yamasaki, T. Yamamoto, M. Inokuchi, S. (1987) CAI System for Acquiring Good Writing Skills Based on the Analysis of Pen Speed, IEICE, J70-D(11), 2071-2076.

[4] Yamamoto M., Yamasaki T., Inokuchi S. (1989). A CAI System for Penmanship Using Calligraphic Skill Knowledge, IEICE Trans. \& Syst. , J72(9), 1493-1500

[5] Hayashi, T., Oda, Y., Sawada, K., Hayashida, Y. (2001). Development of a Game Style Drill System for Learning Kanji Strokes. Transactions of Japanese Society for Information and Systems in Education, 18(1), 7-15

[6] Hayashi, T., Hayashida, Y. (2002) Kanji Master: Kanji Learning System with a Part Structure Assembly Game. Transactions of Japanese Society for Information and Systems in Education, 19(4), 240-245

[7] Shin, J., Sakoe, H. (1999) Stroke Correspondence Search Method for Stroke-Order and Stroke-Number Free On-Line Character Recognition-Multilayer Cube Search, IEICE Trans. \& Syst., J82-D-II(22), 230-239.

[8] Shin, J., Takeda, A. (2004) Character Learning System Using Inter-stroke information, the 8th International Conference on Knowledge-Based Intelligent Information, 165-174, New Zealand

[9] Shin, J., Shimizu, Y. (2012). Learner-Friendly Kanji Learning System with Radical Analysis. International
Journal of Evaluation and Research in Education, 1(1), 1724 